\title{
Review on Synthesis of Thiazole Derivatives from $\alpha$-Halo- ketones and Thiourea or $\mathrm{N}$-Substituted Thiourea
}

Sagar Ramdas Shrimandilkar*

*Department of Chemistry, Sangamner Nagarpalika Arts, D. J. Malpani Commerce and B. N. Sarda Science College, Sangamner, 422605 , Maharashtra, India

\begin{abstract}
Article Info
Volume 7, Issue 4

Page Number: 1-10

Publication Issue :

July-August-2020
\end{abstract}

\section{Article History}

Accepted : 13 Aug 2020

Published : 19 Aug 2020

\section{ABSTRACT}

Thiazole represents important class of heterocyclic compounds. Thiazole and its derivatives have been reported to possess antitubercular, antibacterial, antiinflammatory, anticancer, antifungal activity. Several methods of synthesis of thiazole derivatives have been reported, but most widely used synthetic approach to obtained thiazole derivatives is Hantzsch process. It involved synthesis of thiazole derivative from $\alpha$-halo-carbonyl compounds and thiourea or thiourea derivatives. This review mainly focuses on the research work reported in the scientific literature on the different procedures of synthesis of 2-amino thiazole and their derivatives.

Keywords : Review, thiazole, thiourea, N-substituted thiourea, 2aminothiazole, Hantzsch process.

\section{INTRODUCTION}

The nitrogen and sulphur are important elements in biologically active heterocyclic compounds. The thiazole ring is a structural component of natural compounds such as thiamine, penicillin and carboxylase and its derivatives play an important role in nature and have great broad application in medicinal chemistry. The thiazole ring system is a useful structural motif found in numerous biologically active molecules1-2.The thiazole derivatives have been reported as antitubercular3 antibacterial3-8, anti-inflammatory10-15antifungal4,16, anticancer agents17-22 .
Thiazole ring is synthesized by various methods, but the most widely used synthetic approach to prepare thiazole ring involves the $\alpha$-haloketones with thiourea or thioamides, which is known as Hantzsch process.

Some of the thiazole possessing drugs like thiazolsulfane, sulfathiazole, abafungin, alagebrium, cefepime, cefixime, cefemenoxime, clomethiazole, tazolole, tiabendazole, tiazofurin, meloxicam, fentiazac, etc. The structures of some of these drugs are shown in Fig.01
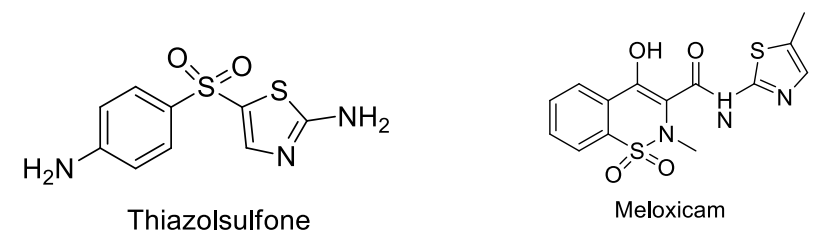

Copyright: ( the author(s), publisher and licensee Technoscience Academy. This is an open-access article distributed under the terms of the Creative Commons Attribution Non-Commercial License, which permits unrestricted noncommercial use, distribution, and reproduction in any medium, provided the original work is properly cited 


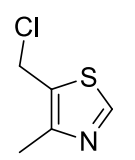

Clomethiazole<smiles>Nc1ccc(S(=O)(=O)Nc2nccs2)cc1</smiles>

Fig. 01

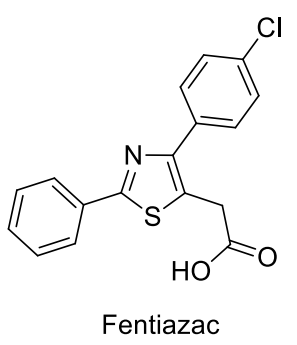

Fentiazac<smiles>[R]C(=O)CBr</smiles>

\section{SYNTHESIS OF THIAZOLE DERIVATIVES}

E. O. Olawode, R. Tandlich, et al. ${ }^{23}$ have reported the synthesis of 2-Chloro-3-[(thiazole-2-yl)amino]-1,4naphthoquinones. In this synthesis they used 2-amino thiazoles, which synthesized from $\alpha$-halo carbonyl compounds and thiourea in ethanol at $70^{\circ} \mathrm{C}$ for 1 hour.<smiles></smiles>

Reagents and conditions: (a) ethanol, $70^{\circ} \mathrm{C}, 1 \mathrm{~h}(\mathrm{~b})$ $\mathrm{K}_{2} \mathrm{CO}_{3}$, DMF, room temperature, $5 \mathrm{~h}$

Mykhaylo A. Potopnyk, Roman Lytvyn, et al. ${ }^{24}$ have synthesized N,O $\pi$-Conjugated 4-Substituted 1,3Thiazole $\mathrm{BF}_{2}$ Complexes, which involved synthesis of 2 - amino thiazole derivatives from $\alpha$-bromo carbonyl compounds and thiourea in ethanol under refluxed condition for 4 hours ${ }^{25}$.

Reagents and conditions: (a) ethanol, reflux, $4 \mathrm{~h}$ (b) pN,N-dimethybenzoylchloride, 1,4-dioxane, TMA, DMAP, reflux, $24 \mathrm{~h}$ (d) $\mathrm{BF}_{3}: \mathrm{Et}_{2} \mathrm{O}, \mathrm{N}, \mathrm{N}-$ diisoproptlyethylamine, Stirring, room temperature, $24 \mathrm{~h}$.

Deepak Mishra, Atiya Fatima, et a ${ }^{25}$ have reported an efficient one pot synthesis of 2-amino thiazole derivatives from $\alpha$-bromo carbonyl compounds and thiourea after stirring in THF at room temperature for 15 minutes.<smiles>[R]c1ccc(C(=O)CBr)cc1[R]</smiles>

Reagents and conditions: (a) THF, stirring at room temperature, 15 Min.

S. S. Kottawar, T. M. Potewar, et al. ${ }^{26}$ have reported the synthesis of 2-aminothiazole derivatives from phenancyl bromides and thiourea or $\mathrm{N}$-substituted thiourea using I2, DMSO at room temperature for 2-3 minutes.<smiles>[R]Nc1nc([Al]CC)cs1</smiles>

Reagents and conditions: (a) I2, DMSO, room temperature, 2-3 min. 
S. Zheng, Q. Zhong et al. ${ }^{27}$ have reported the synthesis of novel thiazole derivatives with good yield in which $\alpha$-bromoketones cyclized with thiourea in ethanol with refluxing condition. The completion of reaction is monitored by TLC.

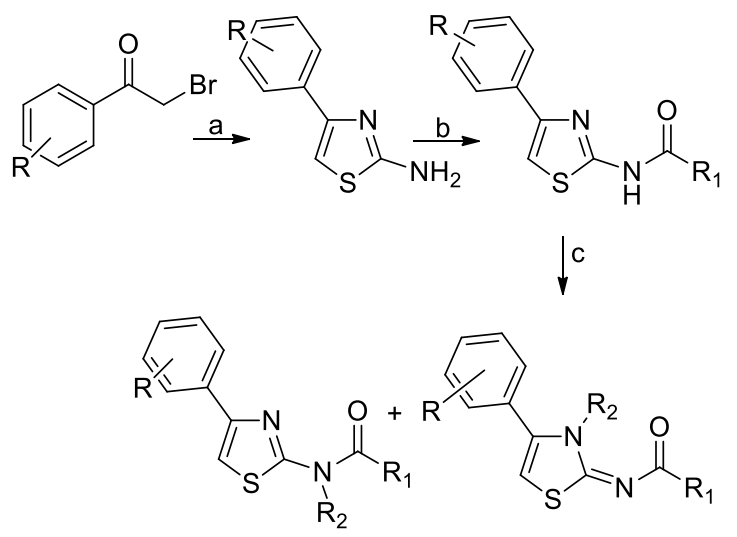

Reagents and conditions: (a) thiourea, ethanol, reflux (b) Method A: R1COOH, DCC, DMAP, $\mathrm{CH}_{2} \mathrm{Cl}_{2}$, Room Temperature; Method B: $\mathrm{R}_{1} \mathrm{COCl}, \mathrm{DMAP}, \mathrm{CH}_{2} \mathrm{Cl}_{2}, 0^{0}$ C to room temperature (c) $\mathrm{NaH}, \mathrm{THF}, 0^{\circ} \mathrm{C}$ to $\mathrm{RT}$; or $\mathrm{MeI}$ or $\mathrm{EtBr} 0^{\circ} \mathrm{C}$ to room temperature.

G. Ramesh, B. Janardhan, et al. ${ }^{28}$ have synthesized 2,4 disubstituted-thiazole derivatives from phenancyl bromides and thiourea or N-substituted thiourea in water under ultrasound at $25^{\circ} \mathrm{C}$. They also reported synthesis of thiazole derivatives with 3-(2Bromoacetyl)coumarins.<smiles>[R]c1ccc(C(=O)CBr)cc1</smiles>

Reagents and Conditions: Water, $\left.\left.\left.25^{\circ} \mathrm{C},()\right)\right)\right)$ )

(Ultrsonication), 10-30 sec.

T. V. Sravanthi, S. L. Manju ${ }^{29}$ have synthesized indol3yl-thiazolo[3,2-a][1,3,5]triazines .In this synthesis phenancyl bromides were prepared from acetophenones .The phenancyl bromides were condensed with thiourea in ethanol at $45^{\circ} \mathrm{C}$ under ultrasounds for 20-35 min.
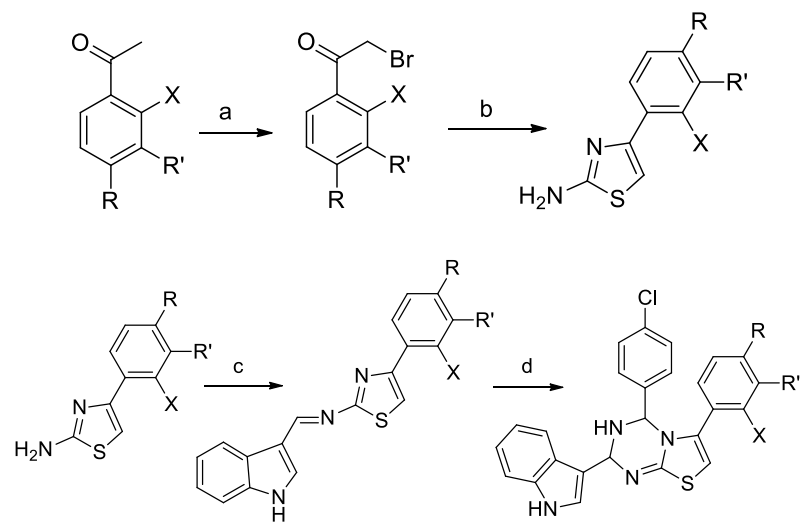

Reagents and conditions: (a) $\mathrm{KBr}-\mathrm{KBrO}_{3}(2: 1)$, dil $\mathrm{HCl}$, Stirr, RT (b) Thiourea, ethanol, US, $45^{\circ} \mathrm{C}, 20-35 \mathrm{~min}$.

(c) Indol-3-Carbaldehyde, ethanol, Warm, 30 min.

(d) p-chlorobenzaldehyde, ammonium acetate, ethanol, reflux.

S. N. Dighe, P. K. Chaskar, et $\mathrm{al}^{30}$ have reported the synthesis of 2-substituted-amino-4-aryl thiazole derivatives in polar solvent DMSO in absence of catalyst under ambient reaction condition within 3040 sec.

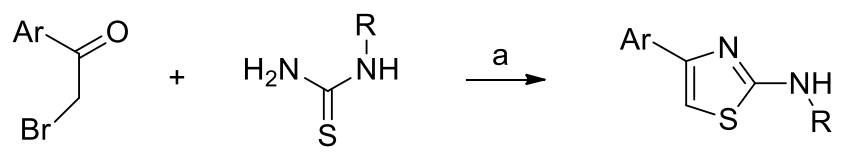

Reagents and conditions: (a) polar solvent-DMSO, room temperature, stirring, 30-40 sec.

Hans-Peter Buchstaller, Uwe Anlauf ${ }^{31}$ have reported the effective procedure for the solution-phase synthesis of 2-aminothiazole.The reaction, work up and separation of 2-aminothiazole was carried out in fully automated fashion by using the Chemspeed ASW2000 automated synthesizer.<smiles>[R]Nc1nc([R3])c([R2])s1</smiles> 
Reagents and conditions: (a) THF or dioxane, $55-70^{\circ} \mathrm{C}$, 8-48 h; (b) Et $3 \mathrm{~N}$; (c) Liquid-liquid extraction.

J. Banothu, K.Vaarlaa, et al. $^{32}$ have synthesized thiazole derivatives from $\alpha$-bromoketones and thiourea or substituted thiourea by using NaF catalyst at room temperature within 1-3 min.<smiles>[R]C(=O)CBr</smiles>

Reagents and conditions: (a) $\mathrm{NaF}, \mathrm{MeOH}$ : $\mathrm{H}_{2} \mathrm{O}$, room temperature, 1-3 min.

S. Rostamizadeh, R. Aryan, et al. ${ }^{33}$ have reported the aqueous $\mathrm{NaHSO}_{4}$ catalyst for regioselective synthesis of 2-aminothiazole derivatives by Hantzsch process.

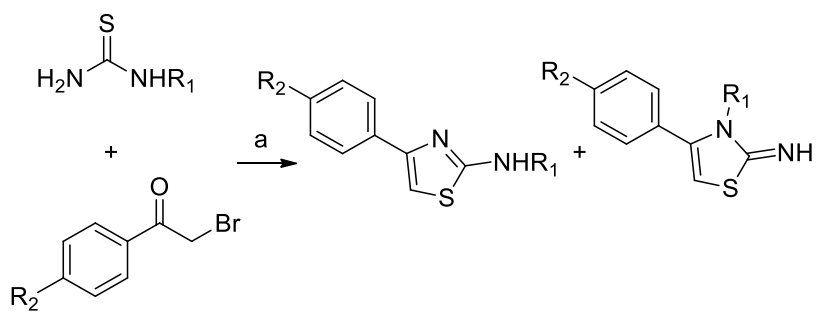

Reagents and Conditions: (a) $\mathrm{H}_{2} \mathrm{O}, \mathrm{NaHSO}_{4}$, room temperature.

T. M. Potewar, S. A. Ingale, et al. ${ }^{34}$ have synthesized 2-aminothiazole derivatives in water at room temperature without catalyst within 1-2 h.<smiles>[Y]Nc1nc([Al]C[Te])cs1</smiles>

Reagent and Conditions: (a) $\mathrm{H}_{2} \mathrm{O}$, room temperature, 1-2 h.

T. M. Potewar, S.A. Ingale, et $\mathrm{al}^{35}$ have reported synthesis of 2,4-disubstituted thiazole derivatives using ionic liquid at room temperature within $10-20$ $\min$.

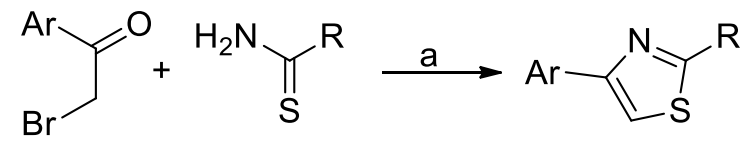

Reagents and conditions :(a) $[$ bbim $] \mathrm{BF}_{4}$, room temperature.

D. Caceres-Castilo, R. M. Carballo, et al. ${ }^{36}$ have synthesized 2-amino-4-arylthiazole derivatives from p-substituted acetophenones and thiourea in presence of iodine under microwave irradiation for $10 \mathrm{~min}$.<smiles>[R]c1ccc(C(C)=O)cc1</smiles>

Reagents and conditions: (a) $\mathrm{I} 2, \mathrm{MW}, 130-150^{\circ} \mathrm{C}, 50$ $\mathrm{W}, 10 \mathrm{~min}$.

\section{III.REFERENCES}

[1] D. Caceres, et al. Tet Lett. , 2012, 53, 3934-3936.

[2] Yan Ping Zhu, J J Yuan., et al., Tetrahedron, 2012, 68,173, 178.

[3] Y. K. Abhale, P. C. Mhaske, IJSRST,2017, 3( 9).

[4] S.K. Bharati, G. Nath, R. Tilak, et al., Eur. J. Med. Chem. 2010, 45, 651-660

[5] M. D. Kinnick, P. Lind, et al., J. Med. Chem. 1995, 38, 4929

[6] K. Tsuji, H. Ishikawa, Bioorg. Med. Chem. Lett. 1994, 4, 1601

[7] Osman SAA, Abdalla AA, Alaib MO. J Pharm Sci. 1983;72:68.

[8] Tandon V K, Yadav D B, et al., Bioorg Med Chem. 2006;14:6120.

[9] Cai P, Kong F, et al., J Nat Prod. 2005;68:1736.

[10] Holla B S, Malini K V, et al., Europ. Journal of Med. Chem. 2003, 38, 313-318.

[11] Kalkhambkar R G, Kulkarni G M, et al., European Journal of Med. Chem. 2007, 42,12721276. 
[12] Haviv, J. D. Ratajczyk, R. W. DeNet, et al., Journal of Med. Chem., vol. 31, no. 9, pp. 17191728, 1988.

[13] F.W. Bell, A. S. Cantrell, M. H“ogberg, et al., Journal of Med. Chem., vol. 38, no. 25, pp. 49294936, 1995.

[14] K. Tsuji and H. Ishikawa, Bioorg. Med. Chem. Lett., 1994 , 4, 1601

[15] B.V. Yang, D.S. Weinstein, et al., J. Med. Chem. 2010, 53, 8241-8251.

[16] Ramírez J, Svetaz L, et al., Eur J Med Chem, 2015, 92, 866.

[17] Ramla MM, Omar MA, et al., Bioorganic and Med. Chem., 2006, 14, 7324-7332.

[18] Gulsory E, Guzeldemirci NU.. European Journal of Med. Chem., 2007, 42, 320-326.

[19] McInnes, C., Drug Discov. Today, 2008, 13(1920), 875-881.

[20] E.L. Luzina, A.V. Popov, Eur. J. Med. Chem., 2009,44, 4944

[21] Cushing, T. D.; Metz, D. P., et al., J. Med. Chem. 2012, 55, 8559-8581.

[22] Romagnoli, R.; Baraldi, P. G.; Salvador, et al., Med. Chem. 2012, 55, 5433-5445.

[23] E. O. Olawode, R. Tandlich et al., Bioorganic and Medicinal Chemistry letters, 2019,29, 1572-1575.

[24] M. A. Potopnyk, R. Lytvyn et al., Journal of Organic Chem. 2018, 83, 1095-1105.

[25] Deepak Mishra, Atiya Fatima, et al. Der Chemica Sinica, 2015,6(8), 14-18.

[26] S. S. Kottawar, T. M. Potewar, et al., Heterocyclic Letters, 2015, 5(2) , 279-283.

[27] S. Zheng, Q. Zhong et al, ACS Medicinal Chemistry letters, 2013,4(20), 191-196.

[28] G. Ramesh, B. Janardhan, et al., Research on Chemical Intermediates, 2015, 41 (11), 80998109.

[29] T. V. Sravanthi, S. L. Manju, Journal of Fluorescence, 2015, 25 (6), 1727-1738.

[30] S. N. Dighe, P. K. Chaskar, et al. ,ISRN Organic Chemistry, 2011, 434613, 6 pp.
[31] Hans-Peter Buchstaller , Uwe Anlauf, Combinatorial Chemistry and High Throughput Screening, 2011,14, 104-108.

[32] J. Banothu, K Vaarlaa, et al.,Chinese Chem. Letters, 2014, 25, 172-175.

[33] S. Rostamizadeh, R. Aryan et al., Monatsh Chem, 2008,139, 1241-1245.

[34] T. M. Potewar, S. A. Ingale, et al., Tetrahedron 2008, 64 5019-5022.

[35] T. M. Potewar, S.A. Ingale, et al., Tetrahedron, 2007, 63, 11066-11069.

[36] D. Caceres-Castilo, R. M. Carballo, et al., Tetrahedron Letters, 2012, 53, 3934-3936.

\section{Cite this article as :}

Sagar Ramdas Shrimandilkar, "Review on Synthesis of Thiazole Derivatives from $\alpha$-Halo- ketones and Thiourea or N-Substituted Thiourea", International Journal of Scientific Research in Science and Technology (IJSRST), Online ISSN : 2395-602X, Print ISSN : 2395-6011, Volume 7 Issue 4, pp. 221-225, July-August 2020. Available at doi $\quad$ : https://doi.org/10.32628/IJSRST207466

Journal URL : http://ijsrst.com/IJSRST207466 